\title{
The transport properties of Dirac fermions in chemical vapour-deposited single-layer graphene
}

\section{Engin Arslan, Şükrü Ardalı, Engin Tıraş, Semih Çakmakyapan \& Ekmel Özbay}

To cite this article: Engin Arslan, Șükrü Ardalı, Engin Tıraș, Semih Çakmakyapan \& Ekmel Özbay (2017) The transport properties of Dirac fermions in chemical vapour-deposited single-layer graphene, Philosophical Magazine, 97:3, 187-200, DOI: 10.1080/14786435.2016.1247994

To link to this article: https://doi.org/10.1080/14786435.2016.1247994

\section{册 Published online: 24 Oct 2016.}

\section{ए马}

Submit your article to this journal $\widetilde{ }$

Џلll Article views: 237

Q View related articles $₫$

View Crossmark data $\asymp$ 


\title{
The transport properties of Dirac fermions in chemical vapour-deposited single-layer graphene
}

\author{
Engin Arslanª, Şükrü Ardalıb, Engin Tıraş̧, Semih Çakmakyapan and Ekmel Özbay ${ }^{\mathrm{a}, \mathrm{d}}$ \\ aDepartment of Physics, Nanotechnology Research Center-NANOTAM, Bilkent University, Ankara, Turkey; \\ ${ }^{b}$ Faculty of Science, Department of Physics, Anadolu University, Eskisehir, Turkey; 'Electrical Engineering \\ Department, University of California, Los Angeles, CA, USA; ${ }^{d}$ Department of Electrical and Electronics \\ Engineering, Bilkent University, Ankara, Turkey
}

\begin{abstract}
The electronic transport properties of Dirac fermions in chemical vapour-deposited single-layer epitaxial graphene on anSiO $/ \mathrm{Si}$ substrate have been investigated using the Shubnikov-de Haas $(\mathrm{SdH})$ oscillations technique. The magnetoresistance measurements were performed in the temperature range between 1.8 and $43 \mathrm{~K}$ and at magnetic fields up to $11 \mathrm{~T}$. The 2D carrier density and the Fermi energy have been determined from the period of the $\mathrm{SdH}$ oscillations. In addition, the in-plane effective mass as well as the quantum lifetime of 2D carriers have been calculated from the temperature and magnetic field dependences of the $\mathrm{SdH}$ oscillation amplitude. The sheet carrier density $\left(1.42 \times 10^{13} \mathrm{~cm}^{-2}\right.$ at $\left.1.8 \mathrm{~K}\right)$, obtained from the lowfield Hall Effect measurements, is larger than that of $2 \mathrm{D}$ carrier density $\left(8.13 \times 10^{12} \mathrm{~cm}^{-2}\right)$. On the other hand, the magnetoresistance includes strong magnetic field dependent positive, non-oscillatory background magnetoresistance. The strong magnetic field dependence of the magnetoresistance and the differences between sheet carrier and 2D carrier density can be attributed to the 3D carriers between the graphene sheet and the $\mathrm{SiO}_{2} / \mathrm{Si}$ substrate.
\end{abstract}

\section{ARTICLE HISTORY}

Received 3 August 2015

Accepted 6 October 2016

\section{KEYWORDS}

B1.Graphene; A1.SdH oscillations; A1.Hall effect

\section{Introduction}

Because of its exceptional properties, including ballistic transport, high current density, high thermal conductivity, chemical inertness, optical transmittance and super hydrophobicity at the nanometer scale, graphene, a one-atom-thick planar sheet of $\mathrm{sp}^{2}$-bonded carbon atoms densely packed in a honeycomb crystal lattice [1-4], has been attracting interest for applications such as solar cells, light-emitting devices, touch screens, photodetectors, ultrafast lasers, membranes, spin valves and high-frequency electronics[5-7].

Different methods are used for graphene synthesis [8]. The first success in the synthesis of monolayer graphene was made by the group of Geim and Novoselov using mechanical exfoliation from bulk graphite [1]. The other mostly used methods are based on the thermal graphitisation of an $\mathrm{SiC}$ surface and by chemical vapour deposition (CVD) using a metallic 
catalyst such as $\mathrm{Ni}, \mathrm{Cu}$ and $\mathrm{Ru}$ [6]. The usages of the graphene in the device applications require the growth of a single- or multi-layer graphene on a suitable substrate. A detailed understanding of the carrier transport properties and scattering mechanisms of a graphene sheet is very important because the material is a potential candidate for being incorporated into the future generation of nano-electronic and nano-photonic devices $[3,4,6]$.

The transport properties of graphene were studied both theoretically [9-11] and experimentally [12-19]. Tiraş et al. [16] published Shubnikov-de Haas ( $\mathrm{SdH}$ ) measurement results for single-layer graphene on a $\mathrm{SiC}$ substrate. They calculate the electronic properties, such as sheet carrier density, 2D carrier density, in-plane effective mass, quantum lifetimes and power loss for single-layer graphene from the SdH oscillations. Zou et al. [14] reported $\mathrm{SdH}$ measurement results for bilayer graphene on $\mathrm{SiO}_{2} / \mathrm{Si}$ substrate. They calculated the effective mass both for the 2D hole and 2D electron gas for a wide range of carrier densities using $\mathrm{SdH}$ oscillations. In the study of Hong et al. [13], they published the results for the transport lifetime and the quantum lifetime in the single-layer graphene sheets that mechanically exfoliated onto $\mathrm{SiO} 2 / \mathrm{Si}$ substrates using $\mathrm{SdH}$ measurements, and suggested carrier mobility limiting scattering mechanisms. However, up to now, there have been no published experimental results for the transport properties that are determined by $\mathrm{SdH}$ measurement in single-layer CVD graphene (SLG) on $\mathrm{SiO}_{2} / \mathrm{Si}$ substrate.

The purpose of the present paper is to study the transport properties of the SLG sheet on $\mathrm{SiO}_{2} / \mathrm{Si}$ substrate. We present the results of the $\mathrm{SdH}$ effect and low-field Hall Effect measurements in p-doped single-layer graphene on $\mathrm{SiO}_{2} / \mathrm{Si}$ substrate. The sheet carrier density, $2 \mathrm{D}$ carrier density, in-plane effective mass, quantum lifetimes of 2D hole gas in a single-layer graphene sheet were determined experimentally. A strong magnetic field dependence of the magnetoresistance results was observed. This behaviour was attributed to the parallel conduction in the $\mathrm{SLG} / \mathrm{SiO}_{2} / \mathrm{Si}$ sample. In the case of single graphene sheet on an $\mathrm{SiO}_{2} / \mathrm{Si}$ substrate, we have a degenerate high-mobility $2 \mathrm{D}$ hole gas in single-layer graphene with a much lower mobility $3 \mathrm{D}$ hole gas between the graphene sheet and $\mathrm{SiO}_{2} / \mathrm{Si}$ substrate.

\section{Samples preparation and measurement details}

In the present study, SLG, which was grown on thin-film copper and subsequently transferred to an $\mathrm{SiO}_{2}(285 \mathrm{~nm}) / \mathrm{Si}$ substrate, was used. The graphene wafers were lithographed by conventional electron-beam lithography into Hall bar geometry with the length and width of $L=1000 \mu \mathrm{m}$ and $w=500 \mu \mathrm{m}$, respectively (Figure $1(\mathrm{a})$ ). The $20 \mathrm{~nm}$ titanium and $100 \mathrm{~nm}$ gold were deposited by an electron beam evaporator, and then followed with the standard lift-off process. The mesa lithography step was performed in order to preserve the active graphene region, while etching the rest of the graphene on the surface with $\mathrm{O}_{2}$ plasma. Interconnect metal lithography was performed using 30/220 nm Ti/Au metal pair. Finally, devices were bonded for measurements.

The layer number of the graphene sample was identified by Raman spectroscopy. Raman spectra were collected by the Jobin Yvon Horiba Raman Spectrometer system at room temperature. As an excitation source, a wavelength of $532 \mathrm{~nm}(2.33 \mathrm{eV})$ from a $\mathrm{He}-\mathrm{Ne}$ excitation laser was applied. The data were collected with a $100 \mathrm{X}$ objective with 0.9 numerical apertures. A slit size of $200 \mathrm{um}$ and a hole size of $1100 \mu \mathrm{m}$ was used throughout the measurements. The magnetoresistance $\left(R_{x x}\right)$ measurements were performed using DC techniques under a static magnetic field up to $11 \mathrm{~T}$ in the temperature range from 1.8 to $43 \mathrm{~K}$ in 


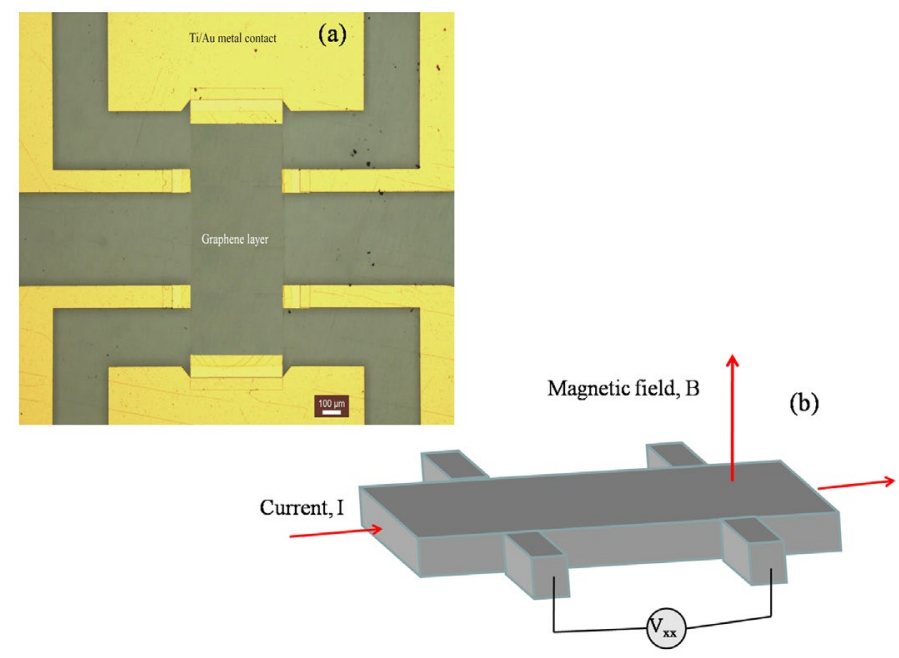

Figure 1. (colour online) (a) Optical image and (b) schematic diagram of the Hall-bar device patterned on an $\mathrm{SLG} / \mathrm{SiO} 2 / \mathrm{Si}$ sample.

the cryogen free superconducting magnet system (Cryogenics Ltd., Model No. J2414) with combination of a constant current source (Keithley 2400) and a nanovoltmeter (Keithley $2182 \mathrm{~A})$. The current flow was in the plane of the $2 \mathrm{D}$ electron gas, and the current $(100 \mu \mathrm{A})$ through the sample was kept low enough to ensure ohmic conditions. Magnetic fields $(B)$ were applied perpendicular to the plane of the samples. In order to calculate the sheet carrier density and Hall mobility as a function temperature, the $R_{x x}$ and Hall resistance $\left(R_{x y}\right)$ were measured as a function of temperature from 1.8 to $260 \mathrm{~K}$ under the static magnetic field of $1 \mathrm{~T}$.

\section{Results and discussion}

\subsection{Raman measurement}

Raman spectroscopy is known to be a powerful tool used to probe the phonon spectrum of graphene. The Raman spectroscopy of graphene can be used to determine the number of graphene layers and stacking order as well as the density of the defects and impurities $[20,21]$. The Raman spectra exhibit a relatively simple structure characterised by two principle bands designated as the $\mathrm{G}$ and $2 \mathrm{D}$ bands, including a third band $\mathrm{D}$ that may also be apparent in spectra when defects within the carbon lattice are present [20,21]. The G band is a sharp band that appears around $1580 \mathrm{~cm}^{-1}$ in the spectrum of graphene $[20,21]$. The $\mathrm{G}$ band position is highly sensitive to the number of layers present in the sample and is one method for determining layer thickness and is based upon the observed position of this band for a sample $[20,21]$. The $\mathrm{D}$ band is known as the disorder-induced band or the defect band. The intensity of the $\mathrm{D}$ band is directly proportional to the level of defects in the sample $[20,21]$. The $2 \mathrm{D}$ band is the second order of the $\mathrm{D}$ band, sometimes called the overtone of the $\mathrm{D}$ band. Recently, they have demonstrated that the shape of the 2D Raman peak may serve as the fingerprint to distinguish mono-, bi- and few-layer graphene [20,21]. In contrast to the $\mathrm{G}$ band position, the $2 \mathrm{D}$ band method depends not only on the peak position but also 


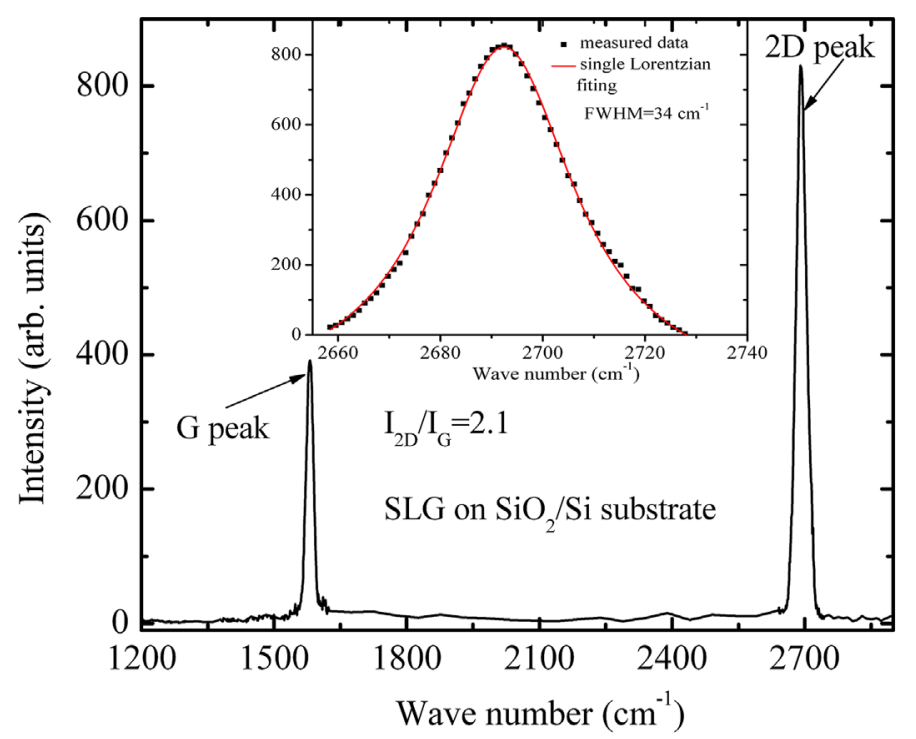

Figure 2. (colour online) Raman spectra of single-layer graphene on $\mathrm{SiO}_{2} / \mathrm{Si}$ substrate.

on the band shape. The band shapes changes with graphene layer number [20]. For single layer graphene, the $2 \mathrm{D}$ band shape is seen as single symmetric peak and can be fitted to a single Lorentzian. With the increasing number of layers, the $2 \mathrm{D}$ band splits into several overlapping modes and requires fitting to two or more Lorentzians. The layer number of the graphene can also be determined by the peak intensity ratio of the $2 \mathrm{D}$ and $\mathrm{G}$ bands.

The Raman spectrum of the graphene layer on $\mathrm{SiO}_{2} / \mathrm{Si}$ substrate is shown in Figure 2 . The Raman spectrum of sample shows 2D peak around $2691 \mathrm{~cm}^{-1}$. We find that the $2 \mathrm{D}$ band is well fitted with a single Lorentzian (inset of Figure 2) with a full with half maximum of $34 \mathrm{~cm}^{-1}$. The $\mathrm{G}$ band position was also observed at $1588 \mathrm{~cm}^{-1}$. On the other hand, Figure 2 shows that no disorder related $D$ peak is observed in the graphene layers. This proves the absence of a significant number of defects. On the other hand, the peak intensity ratio of the $2 \mathrm{D}$ and $\mathrm{G}$ bands was calculated as 2.1 in our graphene layer on the $\mathrm{SiO}_{2} / \mathrm{Si}$ sample. The $\mathrm{I}_{2 \mathrm{D}} / \mathrm{I}_{\mathrm{G}}$ ratio, observation of $\mathrm{G}$ peak at $1588 \mathrm{~cm}^{-1}$, sharp symmetric $2 \mathrm{D}$ peak and lack of $\mathrm{D}$ peak are the confirmation for a high-quality defect-free single-layer graphene sample.

\subsection{Hall mobility and sheet carrier density}

The temperature dependent behaviour of the Hall mobility $\left(\mu_{H}\right)$ and the sheet carrier density $\left(N_{s}\right)$ of carriers was determined from the measured $R_{x x}$ and $R_{x y}$ using equation $[16,18]$;

$$
\begin{gathered}
\mu_{H}=\frac{L}{w} \frac{R_{x y}}{B R_{x x}} \\
N_{s}=\frac{B}{R_{x y} e}
\end{gathered}
$$




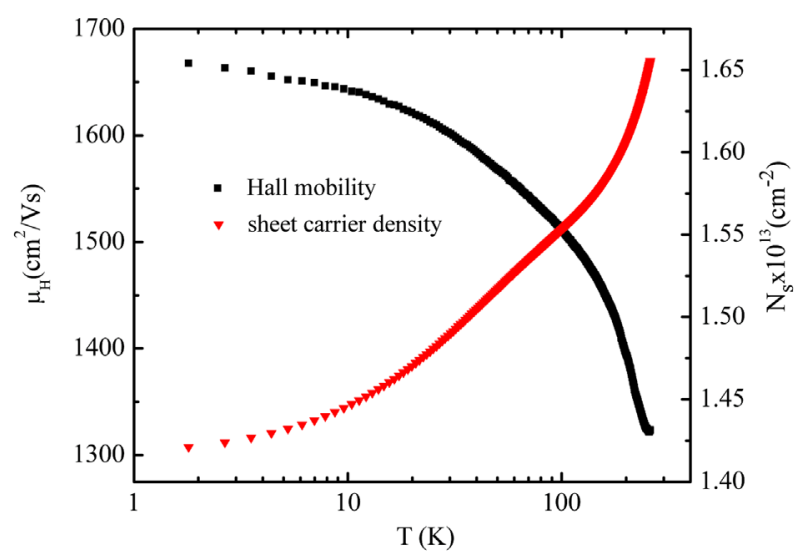

Figure 3. (colour online) Temperature dependence of the Hall mobility $\left(\mu_{H}\right)$ and sheet carrier density $\left(N_{s}\right)$ of hole gas in the $\mathrm{SLG} / \mathrm{SiO}_{2} / \mathrm{Si}$ sample measured at a magnetic field of $1 \mathrm{~T}$.

where $w$ and $L$ are the width and length of the Hall bar, $e$ is the electronic charge and $B$ is the applied magnetic field. $\mu_{H}$ and $N_{s}$ were determined under a static magnetic field of 1.0 $\mathrm{T}$ and temperature between the 1.8 and $260 \mathrm{~K}$. The temperature dependence of the $\mu_{H}$ and $N_{s}$ in the SLG/SiO$/ 2$ si sample is given in Figure 3. From the polarity of the $V_{x y}$, the type of carriers for transport is determined. The results indicate that the current flow in our sample is generated by p-type carriers and that the carrier density increases from $1.42 \times 10^{13} \mathrm{~cm}^{-2}$ at $1.8 \mathrm{~K}$ to $1.65 \times 10^{13} \mathrm{~cm}^{-2}$ at $260 \mathrm{~K}$. This monotonic increment with increasing temperature in the $N_{s}$ values is possibly due to thermally generated carriers. On the other hand, the $\mu_{H}$ of carriers in the $\mathrm{SLG} / \mathrm{SiO}_{2} / \mathrm{Si}$ sample was measured as $1323 \mathrm{~cm}^{2} / \mathrm{Vs}$ at $260 \mathrm{~K}$, and its value increases monotonically with decreasing temperature from 260 to $1.8 \mathrm{~K}$. At $1.8 \mathrm{~K}$, the Hall mobility value reaches $1668 \mathrm{~cm}^{2} / \mathrm{vs}$. This behaviour reflects the $2 \mathrm{D}$ character of the carriers in the single-layer graphene $[16,18]$.

\subsection{SdHoscillations and in-plane effective mass}

The $\mathrm{SdH}$ oscillation in the magnetoresistance of a $2 \mathrm{D}$ system of a single-subband that is occupied is defined by the well-known Lifshitz-Kosevich formula [22], which has been used in both conventional [23-27] and Dirac-like [14,16,17,19] two-dimensional systems,

$$
\frac{\Delta \rho_{x x}}{\rho_{0}}=f\left(\omega_{c} \tau_{q}\right) D(\chi) \exp \left[\frac{-\pi}{\mu_{q} B}\right] \cos \left[\frac{\pi\left(E_{F}-E_{1}\right)}{\hbar \omega_{c}}\right]
$$

where $\Delta \rho_{x x}, \rho_{0}, E_{F}, E_{1}, \omega_{c}\left(e B / m^{*}\right), m^{*}, \mu_{q}$ and $\tau_{q}$ are the oscillatory magnetoresistivity, zero magnetic-field resistivity, Fermi energy, first-subband energy, cyclotron frequency, effective mass, quantum mobility and quantum lifetime of $2 \mathrm{D}$ carriers in the first subband, respectively. The temperature dependence of the envelope function of the oscillations is contained in the function;

$$
D(\chi)=\frac{\chi}{\sinh \chi}
$$


with

$$
\chi=\frac{2 \pi^{2} k_{B} T}{\hbar \omega_{c}}
$$

The longitudinal resistance $\left(R_{x x}\right)$ measurements, as a function of magnetic field for the Hall bar-shaped SLG on $\mathrm{SiO}_{2} / \mathrm{Si}$ samples at various temperatures from 1.8 to $43 \mathrm{~K}$, were performed. Magnetic fields $(B)$ were applied perpendicular to the plane of the samples (Figure 1(b)). The current flow was in the plane of the $2 \mathrm{D}$ electron gas and the constant current $(50 \mu \mathrm{A})$ through the sample was kept low to avoid self-heating. The measured $R_{x x}$ values for SLG on $\mathrm{SiO}_{2} / \mathrm{Si}$ substrate for different temperatures values are shown in Figure 4 . The single periodicity of the SdH oscillations is easily recognised for SLG, as shown in Figure 4. The $\mathrm{SdH}$ oscillations are superimposed on a rising positive non-oscillatory background magnetoresistance. The background values of the $R_{x x}$ show much stronger magnetic field dependence. This behaviour is attributed to the parallel conduction due to the $3 \mathrm{D}$ carriers between the graphene layer and the $\mathrm{SiO}_{2} / \mathrm{Si}$ substrate $[13,28-33]$. In our study, the graphene layers were grown on copper foil by CVD and transferred to the $\mathrm{SiO}_{2} / \mathrm{Si}$ substrate. It is possible for residual copper atoms to remain between graphene layers and $\mathrm{SiO}_{2}$ dielectric materials. This $3 \mathrm{D}$ carrier comes from the residual copper atoms between the graphene layer and $\mathrm{SiO}_{2}$.

In order to reveal the $\mathrm{SdH}$ oscillations from the measured $R_{x x}$ data, the negative second derivative $\left(-\frac{\partial^{2} R_{x x}}{\partial B^{2}}\right)$ with respect to the magnetic field of all the experimental magnetoresistance data was calculated. Figure 5 shows the results of this procedure. This technique does not change the peak position or the period of the oscillations. As seen in Figure 5, the $\mathrm{SdH}$ oscillations extracted from the measured $R_{x x}$ data have well-defined envelopes and they are symmetrical about the horizontal line. In addition, the SdH oscillations have been obtained by subtracting a fitted linear function from the raw experimental data. In the low magnetic field regime $(B<8 \mathrm{~T})$, the $\mathrm{SdH}$ oscillations are relatively small. For this reason,

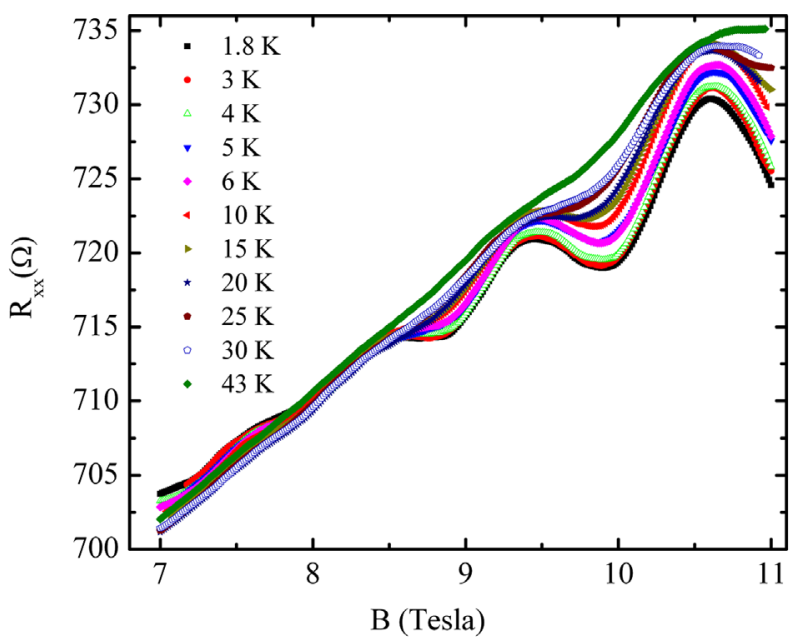

Figure 4. (colour online) Magnetoresistance $\left(R_{x x}\right)$ as a function of the magnetic field measured at various temperature values in the $\mathrm{SLG} / \mathrm{SiO}_{2} / \mathrm{Si}$ sample. 


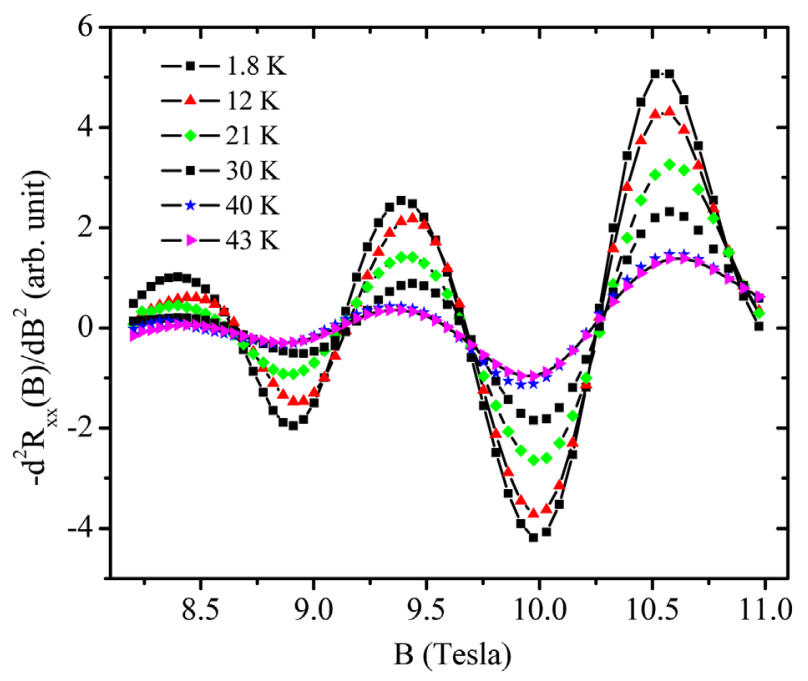

Figure 5. (colour online) Magnetic field dependence of the $\mathrm{SdH}$ oscillations measured at various temperature values in the $\mathrm{SLG} / \mathrm{SiO}_{2} / \mathrm{Si}$ sample. The full curves through the experimental data used to guide the eyes.

we did not show it in the graph. The SdH oscillations damping was measured as a function of ambient temperature. The amplitudes of the oscillations are found to be strongly damped by increasing temperature (Figure 5).

The in-plane effective mass $\left(m^{*}\right)$ of $2 \mathrm{D}$ carriers can be extracted from the temperature dependence of the SdH amplitude at a constant magnetic field using [14,16,23,27],

$$
\frac{A\left(T, B_{n}\right)}{A\left(T_{0}, B_{n}\right)}=\frac{T \sinh \left(\frac{2 \pi^{2} k_{B} m^{*} T_{0}}{\hbar e B_{n}}\right)}{T_{0} \sinh \left(\frac{2 \pi^{2} k_{B} m^{*} T}{\hbar e B_{n}}\right)}
$$

In Equation (6) the amplitudes of the oscillation peaks observed at amagnetic field $B_{n}$ and at the temperatures $T$ and $T_{0}$ are defined with the $A\left(T, B_{n}\right)$ and $A\left(T_{0}, B_{n}\right)$, respectively. As shown in Figure 6, the relative amplitude $A\left(T, B_{n}\right)$ and $A\left(T_{0}, B_{n}\right)$ decreases with increasing temperature in accordance with the usual thermal damping factor [13,14,16,23,27]. By fitting Equation (6) to the experimental data, the $m^{*}$ of $2 \mathrm{D}$ hole gas in SLG on $\mathrm{SiO}_{2} / \mathrm{Si}$ substrate has been determined. The $m^{*}$ of $2 \mathrm{D}$ holes are found to be $0.0599 m_{0}$ at a magnetic field of 9.38 T. A similar fitting process was made for all the oscillation peaks observed in the magnetic-field ranging from 8 to $11 \mathrm{~T}$. In this magnetic field range, the in-plane effective mass of $2 \mathrm{D}$ holes is essentially independent of the magnetic field. Borghi et al. [11] has showed that the Fermi-surface effective mass in single-layer graphene sheets vanishes in the low carrier-density limit. In our study, the higher 2D carrier density $\left(8.13 \times 10^{12} \mathrm{~cm}^{-2}\right)$ in the single-layer graphene caused us to measure the non-vanishing in-plane effective mass values.

\subsection{The $2 D$ hole gas density and Fermi energy}

The period of the $\mathrm{SdH}$ oscillations has been obtained from the plots of the reciprocal magnetic field $\left(1 / B_{n}\right)$, at which the $n$th peak occurs against the peak number $(n)$. In case only one 


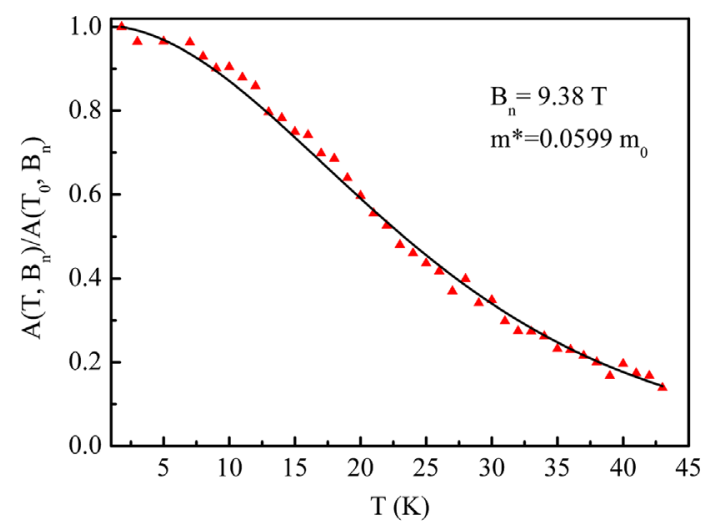

Figure 6. (colour online) Temperature dependence of the normalised amplitude of the SdH oscillation peak measured at a magnetic field of 9.38T. The least-squares fit of Equation (6) to the experimental data is given with a full curve.

subband is occupied by hole gas, the graph of $1 / B_{n}$ vs. $n$ gives a straight line (Figure $7(a)$ ), the slope of which yields the oscillation period, $\Delta(1 / B)$. The Fast Fourier Transformation (FFT) of the $\mathrm{SdH}$ oscillations (inset of Figure 7(a)) confirm that only the first subband is populated. The Fast Fourier spectrum of the SdH oscillations for various temperatures was given in Figure 7(b). As seen in the figure, the oscillation period (hence the carrier density) is found to be essentially independent of temperature. The 2D carrier density $\left(n_{2 D}\right)$ in the SLG/ $\mathrm{SiO}_{2} / \mathrm{Si}$ sample has been calculated using the formula given in Equation (7) $[14,16,23,24,27]$,

$$
\begin{gathered}
n_{2 D}=\frac{g_{v} g_{s} e}{h \Delta\left(\frac{1}{B}\right)} \\
m^{*}=\sqrt{\frac{\pi n_{2 D} \hbar^{2}}{V_{F}^{2}}}=\frac{E_{F}-E_{1}}{V_{F}^{2}}
\end{gathered}
$$

In the equation, $g_{s}, g_{v}$ are the spin and valley degeneracy and taken as two for each other in graphene materials and the $e, \hbar$ and $E_{F}-E_{1}$ are the electric charge, Planck constant and Fermi energy with respect to the first subband energy, respectively. The $n_{2 \mathrm{D}}$ was calculated from the period of the $\mathrm{SdH}$ oscillation. The effective mass and the $\left(E_{F}-E_{1}\right)$ are determined using Equation (8), where the $2 \mathrm{D}$ carrier density is obtained from the $\mathrm{SdH}$ period and $v_{F}=1.1 \times 10^{8} \mathrm{~cm} / \mathrm{s}$ adopted from the literature. The $2 \mathrm{D}$ hole gas density, effective mass and the $\left(E_{F}-E_{1}\right)$ were found to be $8.13 \times 10^{12} \mathrm{~cm}^{-2}, 0.0532 m_{0}$ and $324.19 \mathrm{meV}$ at $1.8 \mathrm{~K}$, respectively, and their values are essentially independent of temperature in the range from 1.8 to $43 \mathrm{~K}$. A good agreement is seen between the effective mass values obtained from temperature-dependent $\mathrm{SdH}$ oscillation $\left(0.0599 m_{0}\right)$ and from the period of the SdH oscillations. However, the sheet carrier density, calculated from the Hall measurements, increases from $1.42 \times 10^{13}$ (at $1.8 \mathrm{~K}$ ) to $1.51 \times 10^{13} \mathrm{~cm}^{-2}$ (at $43 \mathrm{~K}$ ) between the temperature range of 1.8 and $43 \mathrm{~K}$. Increments in the sheet carrier density are explained as the thermal ionisation of the $3 \mathrm{D}$ carriers in the $\mathrm{SiO}_{2} / \mathrm{Si}$ substrate, meanwhile concentration of the $2 \mathrm{D}$ carriers in the 

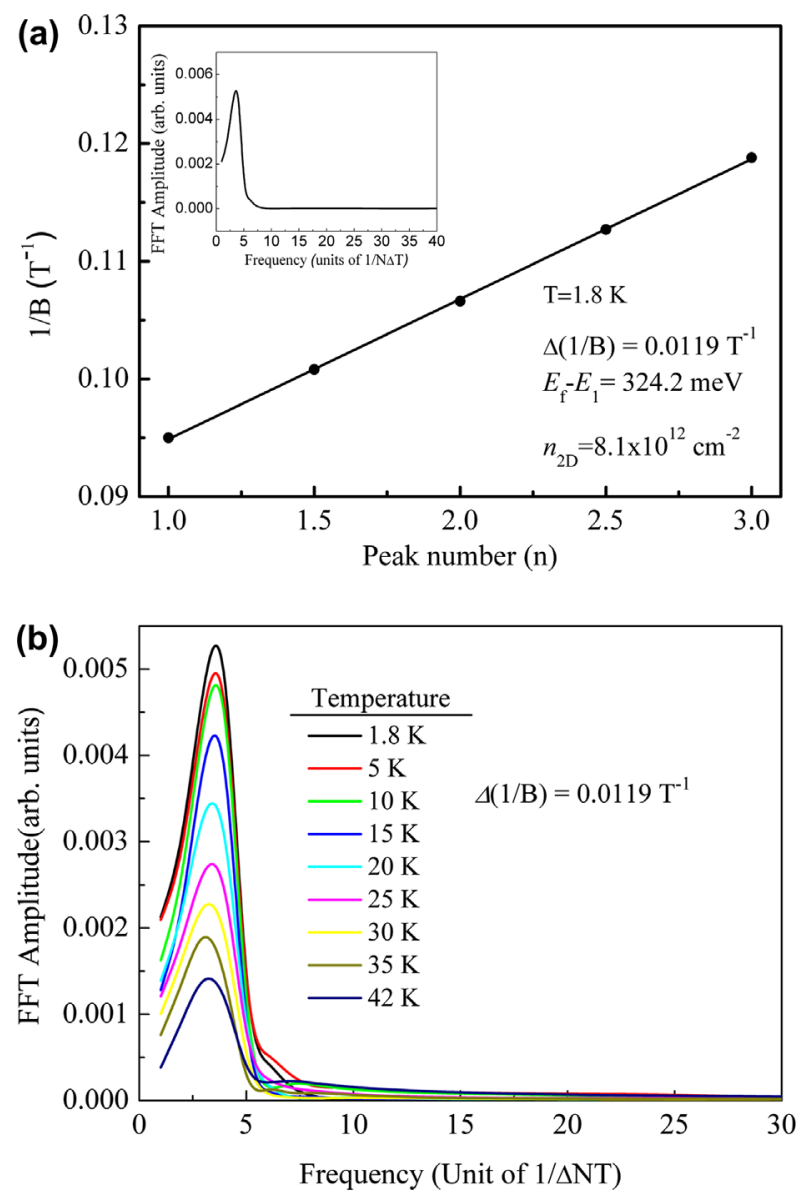

Figure 7. (colour online) (a) The reciprocal magnetic field $\left(1 / B_{n}\right)$ plotted as a function of the oscillation peak number $(n)$. The solid line on the graph is the least-squares fit to the experimental data. The inset shows the fast Fourier spectrum of the SdH oscillations. (b) The FFT of the SdH oscillations measured at various temperatures.

SLG essentially kept constant. In our study, the sheet carrier density is larger than the 2D carrier density calculated from the SdH oscillation. The carrier density differences between two calculations methods are attributed to the bulk $3 \mathrm{D}$ carriers between the graphene sheet and the $\mathrm{SiO}_{2} / \mathrm{Si}$ substrate $[13,28-33]$.

\subsection{The quantum life time of $2 D$ carriers}

The quantum life time $\left(\tau_{q}\right)$ is related to the Landau level broadening through $\Gamma=\hbar / 2 \tau_{q}$ induced by the potentials associated with the carrier-scattering mechanisms [13,34-38]. While $\tau_{q}$ is given by the total scattering rate, in which every scattering event is equally weighted, and is dominated by small-angle scattering, $\tau_{t}$ is weighted by the scattering angle and hence contains no contribution from forward scattering and only a little contribution from small-angle scattering $[13,34,35]$. The relationship between $\tau_{q}$ and the $\tau_{t}$ gives useful information about the nature of the complex scattering scenarios of $2 \mathrm{D}$ electrons (2DEGs) in the system [36,37]. For example, in the modulation-doped GaAs 2DEGs systems, the 

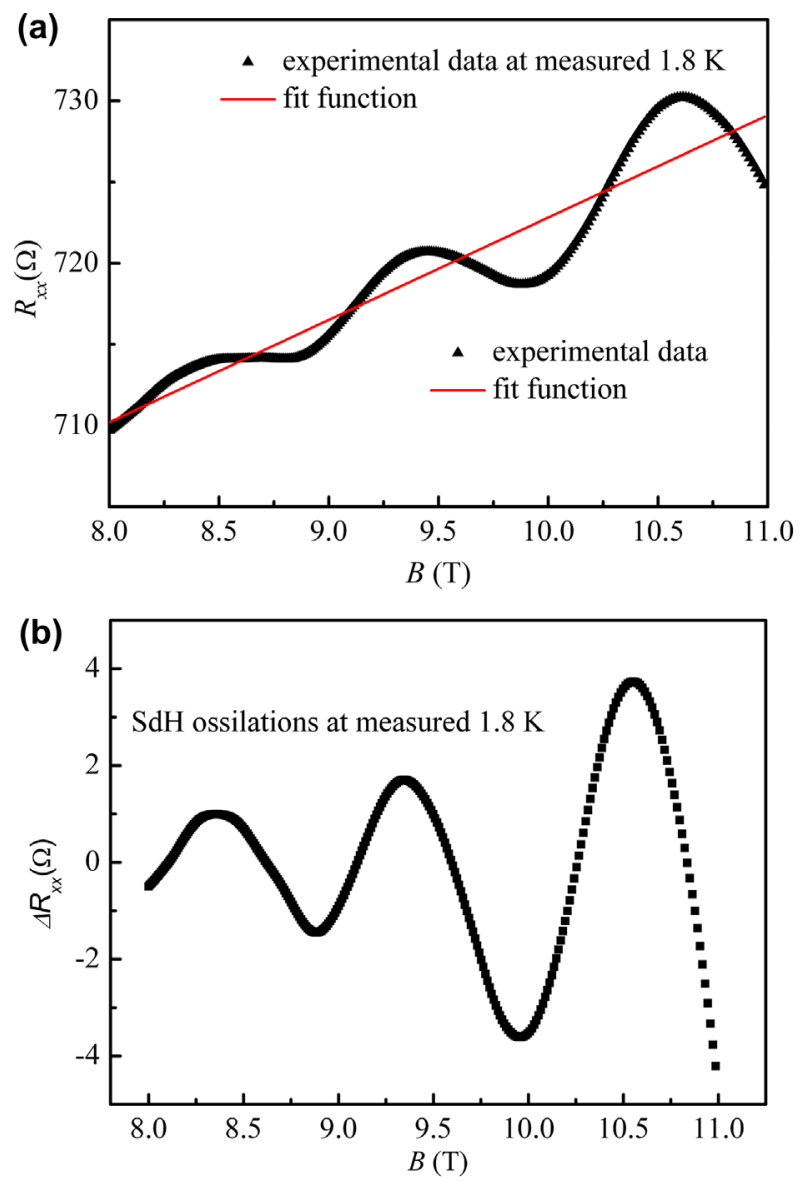

Figure 8. (colour online) (a) Measured magnetoresistance $\left(R_{x x}\right)$ data as a function of magnetic field and linear function that fitted to the experimental data $R_{x x}$ and (b) $\mathrm{SdH}$ oscillations after subtractions of the linear function from the measured $R_{x x}$ data.

long-range nature of the scattering potential of the remote ionised impurity scattering usually results in the scattering time ratio $\tau_{t} / \tau_{q}>>1$. On the other hand, short-range scattering from an alloy disorder potential results in $\tau_{t} / \tau_{q} \sim 1$ [36-38]. However, in the system, when alloy scattering and ionised impurity scattering are simultaneously present, a more complicated behaviour for $\tau_{t} / \tau_{q}$ would be expected.

The $\tau_{q}$ and $\tau_{t}$ are determined from the SdH oscillations and the low-field Hall Effect measurements $[13,16,34]$. The $\tau_{q}$ is one of the factors that control the envelope of the $\mathrm{SdH}$ oscillations. As a consequence, the quantum lifetime, and hence the quantum mobility $\left(\mu_{q}=e \tau_{q} / m^{*}\right)$ ares determined from the magnetic field dependence of the SdH oscillations amplitudes at any given constant temperature using known effective mass values $[13,16,34]$,

$$
\ln \left(\frac{A\left(T, B_{n}\right) \sinh \chi}{\chi}\right)=C-\frac{\pi m^{*}}{e \tau_{q}} \frac{1}{B_{n}}
$$




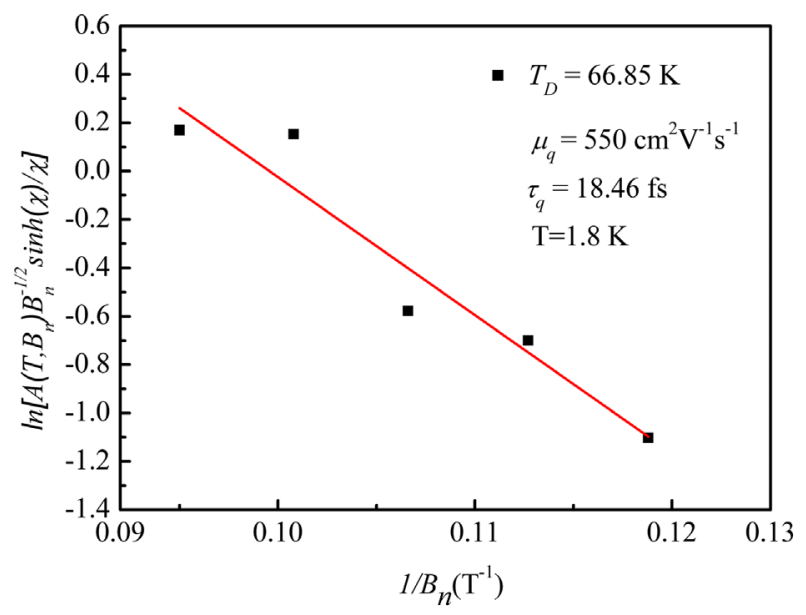

Figure 9. (colour online) Dingle plots of the SdH oscillations for obtaining the quantum lifetime of the $2 \mathrm{D}$ hole gas in the $\mathrm{SLG} / \mathrm{SiO}_{2} / \mathrm{Si}$ sample. The least-squares fit of Equation (9) to the experimental data is given with full straight lines.

The equation given above is called a Dingle plot, where $A\left(T, B_{n}\right), \omega_{c}$ and $C$ are the amplitude of the $n$th oscillation peak at temperature $T$ and magnetic field $B_{n}$, cyclotron frequency and a constant, respectively. The $\chi$ is defined in Equation (5). In order to calculate Dingle temperature, quantum mobility and quantum lifetime from the Dingle plot, we calculated the $A\left(T, B_{n}\right)$ of the $\mathrm{SdH}$ oscillation from the raw data. As shown in Figure 8(a) and (b), firstly, a linear function was fitted to the measured $R_{x x}$ values and subtracted from the $R_{x x}$ and obtained $\mathrm{SdH}$ oscillations $\left(A\left(T, B_{n}\right)\right)$.

Figure 9 shows the Dingle plots for the $\mathrm{SLG} / \mathrm{SiO}_{2} / \mathrm{Si}$ sample at $1.8 \mathrm{~K}$. There is a good agreement between the experimental data and the straight line described by Equation (9). The linear behaviour of the experimental data indicates that the $\tau_{q}$ is independent of the magnetic field as assumed in Equation (9). The quantum lifetime is obtained from the slope of the Dingle plot using the values of $m^{*}$ that are determined from the temperature dependence of the SdH oscillations. The Dingle temperature, quantum mobility and quantum lifetime from the Dingle plot are determined at $1.8 \mathrm{~K}$ as $66.8 \mathrm{~K}, 550 \mathrm{~cm}^{2} / \mathrm{Vs}$ and $18.46 \mathrm{fs}$, respectively. The measured values of $T_{D}$ and $\mu_{q}$ are all independent of the magnetic fields and temperature in our range of measurements. In our study, the $\tau_{t}$ value is calculated from the low-field Hall Effect measurements as $56.80 \mathrm{fs}$. The $\tau_{t} / \tau_{q}$ ratio is obtained as 3.08. The transport lifetime to quantum lifetime ratio found for SLG on $\mathrm{SiO}_{2} / \mathrm{Si}$ substrate is consistent with the previously published data [13].The scattering from the $3 \mathrm{D}$ charges, which stay between the graphene and the substrate and the source of the parallel conduction, limited the mobility of the $2 \mathrm{D}$ carriers in the single-layer graphene on the $\mathrm{SiO}_{2} / \mathrm{Si}$ substrate. Recently, a systematic comparison between $\tau_{t}$ and $\tau_{q}$ has been made in the single-layer graphene sheets that mechanically exfoliated onto $\mathrm{SiO}_{2} / \mathrm{Si}$ substrates by Hong et al. [13]. In their publications, they suggested that the mobility in the single-layer graphene on $\mathrm{SiO}_{2} / \mathrm{Si}$ samples is limited by scattering from charges staying within $2 \mathrm{~nm}$ of the graphene sheet. 


\section{Conclusions}

In the present study, the 2D carrier density, effective mass, quantum lifetime, the sheet carrier density and the transport mobility of $2 \mathrm{D}$ hole gas in the $\mathrm{SLG} / \mathrm{SiO}_{2} / \mathrm{Si}$ sample have been determined by $\mathrm{SdH}$ oscillations and low-field Hall effect measurements. The sheet carrier density is larger than that of the 2D carrier density calculated from the $\mathrm{SdH}$ oscillations. The differences between the sheet carrier density and 2D carrier density are attributed to the $3 \mathrm{D}$ carriers between the $\mathrm{SL}$ graphene and the $\mathrm{SiO}_{2} / \mathrm{Si}$ substrate. The transport lifetime to the quantum lifetime ratio found for $\mathrm{SLG}$ on $\mathrm{anSiO}_{2} / \mathrm{Si}$ substrate is 3.08 .

\section{Acknowledgements}

One of the authors (E.O.) acknowledges partial support from the Turkish Academy of Sciences.

\section{Disclosure statement}

No potential conflict of interest was reported by the authors.

\section{Funding}

This work is supported by the European Union under the projects EU-METAMORPHOSE, EU-PHOREMOST, EU-PHOME, and EU-ECONAM, and TUBITAK [project number 105E066], [project number 105A005], [project number 106E198], and [project number 106A017].

\section{References}

[1] K.S. Novoselov, A.K. Geim, S.V. Morozov, D. Jiang, Y. Zhang, S.V. Dubonos, I.V. Grigorieva, A.A. Firsov, Electric field effect in atomically thin carbon films, Science 306 (2004), pp. 666-669.

[2] K.S. Novoselov, A.K. Geim, S.V. Morozov, D. Jiang, M.I. Katsnelson, I.V. Grigorieva, S.V. Dubonos, and A.A. Firsov, Two-dimensional gas of massless Dirac fermions in graphene, Nature 438 (2005), pp. 197-200.

[3] A.H. Castro Neto, F. Guinea, N.M.R. Peres, K.S. Novoselov, and A.K. Geim, The electronic properties of graphene, Rev. Mod. Phys. 81 (2009), pp. 109-162.

[4] D.R. Cooper, B. D’Anjou, N. Ghattamaneni, B. Harack, M. Hilke, A. Horth, N. Majlis, M. Massicotte, E. Whiteway, L. Vandsburger and V. Yu. Experimental review of graphene, ISRN Condens. Matter Phys. 2012 (2012), 56p, Article ID 501686.

[5] L. Vicarelli, M.S. Vitiello, D. Coquillat, A. Lombardo, A.C. Ferrari, W. Knap, M. Polini, V. Pellegrini, and A. Tredicucci, Graphene field-effect transistors as room-temperature terahertz detectors, Nat. Mater. 11 (2012), pp. 865-871.

[6] F. Bonaccorso, A. Lombardo, T. Hasan, Z. Sun, L. Colombo, and A.C. Ferrari, Production and processing of graphene and $2 d$ crystals, Mater. Today 15 (2012), pp. 564-589.

[7] T. Otsuji, S.A.B. Tombet, A. Satou, H. Fukidome, M. Suemitsu, E. Sano, V. Popov, M. Ryzhiiand, and V. Ryzhii, Graphene-based devices in terahertz science and technology, J. Phys. D: Appl. Phys. 45 (2012), 303001 (9pp).

[8] F. Bonaccorso, Z. Sun, T. Hasan, and A.C. Ferrari, Graphene photonics and optoelectronics, Nat. Photonics 4 (2010), pp. 611-622.

[9] V.P. Gusynin and S.G. Sharapov, Magnetic oscillations in planar systems with the Dirac-like spectrum of quasiparticle excitations. II. Transport properties, Phys. Rev. B 71 (2005), 125124 (8pp).

[10] E.H. Hwang, S. Adam, and S. Das Sarma, Carrier transport in two-dimensional graphene layers, Phys. Rev. Lett. 98 (2007), 186806 (4pp). 
[11] G. Borghi, M. Polini, R. Asgari, and A.H.M. Donald, Fermi velocity enhancement in monolayer and bilayer graphene, Solid State Commun. 149 (2009), pp. 1117-1122.

[12] W.A. de Heer, C. Berger, X. Wu, P.N. First, E.H. Conrad, X. Li, T. Li, M. Sprinkle, J. Hass, M.L. Sadowski, M. Potemski, and G. Martinez, Epitaxial graphene, Solid State Commun. 143 (2007), pp. 92-100.

[13] X. Hong, K. Zou, and J. Zhu, Quantum scattering time and its implications on scattering sources in graphene, Phys. Rev. B 80 (2009), 241415(R)(4pp).

[14] K. Zou, X. Hong, and J. Zhu, Effective mass of electrons and holes in bilayer graphene: Electronhole asymmetry and electron-electron interaction, Phys. Rev. B 84 (2011), 085408 (6pp).

[15] U. Zeitler, A.J.M. Giesbers, H.J. van Elferen, E.V. Kurganova, A. McCollam, and J.C. Maan, Magneto-transport in the zero-energy Landau level of single-layer and bilayer graphene, J. Phys: Conf. Ser. 334 (2011), 012035 (6pp).

[16] E. Tiras, S. Ardali, T. Tiras, E. Arslan, S. Cakmakyapan, O. Kazar, J. Hassan, E. Janzén, and E. Ozbay, Effective mass of electron in monolayer graphene: Electron-phonon interaction, J. Appl. Phys. 113 (2013), 043708 (8pp).

[17] A.M.R. Baker, J.A. Alexander-Webber, T. Altebaeumer, S.D. McMullan, T.J.B.M. Janssen, A. Tzalenchuk, S. Lara-Avila, S. Kubatkin, R. Yakimova, C.-T. Lin, L.-J. Li, and R.J. Nicholas, Energy loss rates of hot Dirac fermions in epitaxial, exfoliated, and CVD graphene, Phys. Rev. B 87 (2013), 045414 (6pp).

[18] E. Arslan, S. Çakmakyapan, Ö. Kazar, S. Bütün, S.B. Lişesivdin, N.A. Cinel, G. Ertaş, Ş. Ardalı, E. Tiraş, J. Ul-Hassan, E. Janzén, and E. Özbay, SiC Substrate effects on electron transport in the epitaxial graphene layer, Electron. Mater. Lett. 10 (2014), pp. 387-391.

[19] J. Huang, J.A. Alexander-Webber, T.J.B.M. Janssen, A. Tzalenchuk, T. Yager, S. Lara-Avila, S. Kubatkin, R.L. Myers-Ward, V.D. Wheeler, D.K. Gaskill, and R.J. Nicholas, Hot carrier relaxation of Dirac fermions in bilayer epitaxial graphen, J. Phys.: Condens. Matter 27 (2015), 164202 (7pp).

[20] A.C. Ferrari, J.C. Meyer, V. Scardaci, C. Casiraghi, M. Lazzeri, F. Mauri, S. Piscanec, D. Jiang, K.S. Novoselov, S. Roth, and A.K. Geim, Raman spectrum of graphene and graphene layers, Phys. Rev. Lett. 97 (2006), 187401 (4pp).

[21] R. Beams, L.G. Cançado, and L. Novotny, Raman characterization of defects and dopants in graphene, J. Phys.: Condens. Matter 27 (2015), 083002 (26pp).

[22] I.M. Lifshitz and A.M. Kosevich, Theory of magnetic susceptibility in metals alt low temperatures, Sov. Phys. - JETP 2 (1956), pp. 636-645.

[23] T. Ando, A.B. Fowler, and F. Stern, Electronic properties of two-dimensional systems, Rev. Mod. Phys. 54 (1982), pp. 437-673.

[24] I. Lo, W.C. Mitchel, R.E. Perrin, R.L. Messham, and M.Y. Yen, Two-dimensional electron gas in GaAs/Al $l_{1-x} G a_{x}$ As heterostructures: Effective mass, Phys. Rev. B 43 (1991), pp. 11787-11790.

[25] J.J. Harris, J.M. Lagemaa, S.J. Battersby, C.M. Hellon, C.T. Foxon, and D.E. Lacklison, Subband populations and the spatial distribution of electrons in $\mathrm{GaAs} /(\mathrm{Al}, \mathrm{Ga})$ As modulation-doped quantum wells, Semicond. Sci. Technol. 3 (1988), pp. 773-780.

[26] S. Elhamri, A. Saxler, W.C. Mitchel, C.R. Elsass, I.P. Smorchkova, B. Heying, E. Haus, P. Fini, J.P. Ibbetson, S. Keller, P.M. Petroff, S.P. DenBaars, U.K. Mishra, and J.S. Speck, Persistent photoconductivity study in a high mobility AlGaN/GaN heterostructure, J. Appl. Phys. 88 (2000), pp. 6583-6588.

[27] A. Bayrakl1, E. Arslan, T. Fırat, S. Özcan, Ö. Kazar, H. Çakmak, and E. Özbay, Magnetotransport study on AlInN/(GaN)/AlN/GaN heterostructures, Phys. Status Solidi (A) 209 (2012), pp. 11191123

[28] E.F. Schubert and K. Ploog, Electron subband structures in selectively doped $n-A I_{x} G a_{1-x} A s / G a A s$ Heterostructures, IEEE Trans. Electron Devices 32 (1985), pp. 1868-1873.

[29] M.J. Kane, N. Apsley, D.A. Anderson, L.L. Taylor, and T. Kerr, Parallel conduction in GaAs/Al $\mathrm{Ga}_{1-}$ ${ }_{x}$ As modulation doped heterojunctions, J. Phys. C: Solid State Phys. 18 (1985), pp. 5629-5636.

[30] F.F. Fang, T.P. Smith III, and S.L. Wright, Landau-level broadening and scattering time in modulation doped GaAs/AlGaAs heterostructures, Surf. Sci. 196 (1988), pp. 310-315.

[31] S. Luryi and A. Kastalsky, Anomalous photomagnetoresistance effect in modulation-doped AlGaAs/GaAs heterostructures, Appl. Phys. Lett. 45 (1984), pp. 164-167. 
[32] S.J. Battersby, F.M. Selten, J.J. Harris, and C.T. Foxon, Magnetoresistance effect in AlGaAs/GaAs two-dimensional electron gas structures at room temperature, Solid-State Electron. 31 (1988), pp. 1083-1088.

[33] M. van der Burgt, V.C. Karavolas, F.M. Peeters, J. Singleton, R.J. Nicholas, F. Herlach, J.J. Harris, M. van Hove, and G. Borghs, Magnetotransport in a pseudomorphic GaAs $/ G a_{0.8} I n_{0.2} A s /$ $\mathrm{Ga}_{0.75} A l_{0.25}$ As heterostructure with a Si $\delta$-doping layer, Phys. Rev. B 52 (1995), pp. 12218-12231.

[34] P.T. Coleridge, Small-angle scattering in two-dimensional electron gases, Phys. Rev. B 44 (1991), pp. 3793-3801.

[35] S. Das, Sarma and Frank Stern, single-particle relaxation time versus scattering time in an impure electron gas, Phys. Rev. B 32 (1985), pp. 8442-8447.

[36] J.P. Harrang, R.J. Higgins, R.K. Goodall, P.R. Jay, M. Laviron, and P. Delescluse, Quantum and classical mobility determination of the dominant scattering mechanism in the two-dimensional electron gas of an AlGaAs/GaAs heterojunction, Phys. Rev. B 32 (1985), pp. 8126-8135.

[37] A. Gold, Scattering time and single-particle relaxation time in a disordered two-dimensional electron gas, Phys. Rev. B 38 (1988), pp. 10798-10811.

[38] S. Syed, M.J. Manfra, Y.J. Wang, R.J. Molnar, and H.L. Stormer, Electron scattering in AlGaN/ GaN structure, Appl. Phys. Lett. 84 (2004), pp. 1507-1509. 